\title{
Intra-arterial versus intra-venous thrombolysis within and after the first 3 hours of stroke onset
}

Srivastava Padma1, Moonis Majaz

1Department of Neurology, AlIMS, New Delhi, India

2Department of Neurology, UMASS, Worcester, USA

Submitted: 13 August 2009

Accepted: 23 September 2009

Arch Med Sci 2010; 6, 3: 303-315

DOI: 10.5114/aoms.2010.14248

Copyright $\odot 2010$ Termedia \& Banach

\begin{abstract}
The NINDS trial demonstrated for the first time the effectiveness of intravenous thrombolysis in improving outcome after acute ischemic stroke. The absolute benefit of this intervention was $11-13 \%$ greater chance of being normal or near normal (MRS $\leq 1)$ at 3 months. However, if patients with severe stroke were considered (NIHSS $\geq 20$ ), the absolute benefit dropped to $5-6 \%$, indicating that IV thrombolysis may not be as effective for large vessel occlusion. This observation was further supported by TCD studies that clearly demonstrated that large artery occlusions had a recanalization rate of $13-18 \%$ with IV rt-PA. Intra-arterial thrombolysis achieves recanalization rates of $60-70 \%$. Since tissue viability is clearly important, it is time to stop defining rigid time windows and if there is a large penumbra (20-50\%) and the occlusion is in a large artery, there exists a logic and a growing evidence to consider either bridge therapy or direct intra-arterial therapy.
\end{abstract}

Key words: intra-arterial, intravenous, ischemic stroke, re-canalization, thrombolysis.

\section{Introduction}

The range of treatments available for acute ischemic stroke (AIS) has expanded considerably in recent years. Dynamic advances in neuroimaging techniques and improved understanding of the pathophysiology of stroke have changed the nihilistic approach of the past to one of hope, resolve and commitment. In general, the timing of various therapeutic interventions is limited to the evolution of the ischemic process. There is a great deal of emerging evidence that may suggest that the duration of the ischemic penumbra varies considerably $[1,2]$. This may depend on factors such as the underlying mechanisms of ischemia and the rate of recanalization. There is evidence that the ischemic penumbra may persist for up to $48 \mathrm{~h}$ after onset, but in some cases it may be $3 \mathrm{~h}$ or even less. The survival of the bulk of ischemic tissue is dependent on early energy failure and ionic imbalances, and smaller volumes are dependent on neurotoxic, inflammatory processes and on apoptosis.

\section{Review}

Thrombolytic therapy is an inherently attractive treatment for AIS based on known pathologic and angiographic substrates of ischemic cerebrovascular disease. Most ischemic strokes are the direct consequence of atherothrombosis or a thromboembolism of a cerebral or precerebral
Corresponding author: M.V. Padma Srivastava Department of Neurology AlIMS, New Delhi, 110029 Phone: 9868398261

E-mail:

vasanthapadma123@gmail.com 
artery. The critical event is usually the formation of an acute thrombus $[3,4]$. The basis for thrombolytic treatment is to achieve rapid arterial recanalization with a relatively safe agent soon enough to improve patient outcome.

\section{Intra-venous thrombolysis}

Prompt reperfusion strategies such as intravenous (IV) recombinant tissue plasminogen activator (rTPA) have provided clinicians with a potent weapon in the armamentarium against AIS, but with clear limitations [5]. The evidence for efficacy is strong and fulfils level I criteria. Cumulative evidence from all trials of TPA within the 3-h time window gives a relative risk reduction of $44 \%$, absolute risk reduction (ARR) of $13 \%$ and number needed to treat (NNT) to save one person from death or disability about 7 [5]. This is a powerful biologic effect and its extent is often under-recognized. To counter this, there is only a modest and non-significant increase in mortality, but an approximately 3 -fold increase in intra-cranial hemorrhage $(\mathrm{ICH})[5]$.

However, only a very small percentage of patients with AIS receive IV rTPA, partly related to the very narrow therapeutic time window for intervention, and multiple, stringent exclusion criteria. Furthermore, IV rTPA results in recanalization in approximately $50 \%$ of patients. Patients treated within 90 min did better than those treated after 90 min within the $3-\mathrm{h}$ window [6]. This difference was significant and suggests that earlier treatment is associated with a better outcome. When analyzed by stroke severity, the magnitude of benefit declined with increasing NIHSS scores. Patients with NIHSS scores $>20$ had a $\leq 6 \%$ ARR in achieving $m R S<1$ at 90 days as against a 13\% ARR in patients with NIHSS scores of $<20$ [5]. This suggests that patients with large vessel occlusions with a high clot burden, as would be expected with the internal carotid artery (ICA), main stem middle cerebral artery (M1), or basilar artery (BA), are less

Table I. Percentage of patients $(n=320)$ in the 91 to 180-min subgroups with a specific baseline National Institutes of Health Stroke Scale (NIHSS) score*

\begin{tabular}{|lcc|}
\hline $\begin{array}{l}\text { Baseline } \\
\text { NIHSS score }\end{array}$ & $\begin{array}{c}\text { tPA-Treated } \\
\text { patients, } \\
\%(n=153)\end{array}$ & $\begin{array}{c}\text { Patients } \\
\text { given placebo, } \\
\%(n=167)\end{array}$ \\
\hline $0-5$ & 19.0 & 4.2 \\
\hline $6-10$ & 24.2 & 27.5 \\
\hline $11-15$ & 17.0 & 21.0 \\
\hline $16-20$ & 21.6 & 19.8 \\
\hline$>20$ & 8.3 & 27.5 \\
\hline
\end{tabular}

tPA - tissue plasminogen activator, *From Marler et al. likely to improve with the present accepted and FDA-approved IV thrombolysis strategy. NINDS unfortunately did not have vascular imaging, and had a relatively low NIHSS, in the group treated between 91-180 $\min (\leq 15=\mathrm{tPA}$ arm $=60.2 \%$; placebo $=52.7 \%$; $\geq$ tPA arm $=39.9 \%$; placebo arm $=47.3 \%$ ) (Table I), suggesting that even the large vessel strokes in NINDS were probably branch occlusions and not main middle cerebral artery (MCA), internal carotid artery (ICA) or basilar artery occlusions [7]. Patients treated from 91 to $180 \mathrm{~min}$ after stroke onset had far less severe strokes than the control (placebo) group. More tPA-treated patients had mild strokes (15\% difference), and fewer had severe strokes (10\% difference). Those differences are significant in absolute terms, but their actual significance becomes apparent when the importance of the graph from the TOAST study (Trial of Org 10172 in Acute Stroke Treatment) is fully appreciated [8]. The graph plots the probability of an excellent outcome of an untreated stroke patient against the baseline NIHSS score. The curves are flat at high baseline NIHSS scores above 20, and stroke patients with a baseline NIHSS score of over 20 have a low probability of an excellent outcome (<10\% favorable outcome rate). While this does not automatically translate to a better outcome with intra-arterial thrombolysis in patients with NIHSS scores > 20 (who may [but not necessarily] have large vessel occlusions and hence a more severe NIHSS score), the observations suggest that mere IV thrombolysis may not be effective in this group of patients.

There has been a relative lack of efficacy of IV rTPA when given after $3 \mathrm{~h}$ of stroke (European Cooperative Acute Stroke Studies [ECASS I and ECASS II]; Alteplase Thrombolysis for Acute Noninterventional Therapy in Ischemic Stroke [ATLANTIS]) [9-12]. A high rate of symptomatic brain hemorrhage was noted in ATLANTIS. A metaanalysis, however, showed benefit from IV therapy up to at least $4.5 \mathrm{~h}$. The recently published results of the ECASS III study confirm the advantage of giving IV rTPA up to $4.5 \mathrm{~h}$ after stroke onset. ECASS III tested the efficacy and safety of alteplase administered between 3 and $4.5 \mathrm{~h}$ after the onset of stroke [13].

Patients were randomly assigned in a $1: 1$ double-blind fashion to receive treatment with intravenous alteplase $(0.9 \mathrm{mg}$ per $\mathrm{kg}$ of body weight) or placebo. The primary end point was disability at 90 days, dichotomized as a favorable outcome (a score of 0 or 1 on the modified Rankin scale, which has a range of 0 to 6 , with 0 indicating no symptoms at all and 6 indicating death) or an unfavorable outcome (a score of 2 to 6 on the modified Rankin scale). The secondary end point was a global outcome analysis of four neurologic 
and disability scores combined. Safety end points included death, symptomatic intracranial hemorrhage, and other serious adverse events. Out of a total of 821 patients in the study, 418 randomly assigned to the rTPA group and 403 to the placebo group, median time for the administration of rTPA was 3 h 59 min. More patients had a favorable outcome with rTPA than with placebo $(52.4 \%$ vs. $45.2 \%$; odds ratio $1.34 ; 95 \%$ confidence interval [CI] 1.02 to $1.76, p=0.04)$. In the global analysis, the outcome was also improved with rTPA as compared with placebo (odds ratio $1.28,95 \% \mathrm{Cl} 1.00$ to 1.65 , $p<0.05)$. The incidence of intracranial hemorrhage was higher with rTPA than with placebo (for any intracranial hemorrhage, $27.0 \%$ vs. $17.6 \%$, $p=0.001$; for symptomatic intracranial hemorrhage, $2.4 \%$ vs. $0.2 \% ; p=0.008$ ). Mortality did not differ significantly between the rTPA and placebo groups (7.7\% and $8.4 \%$, respectively; $p=0.68$ ). There was no significant difference in the rate of other serious adverse events [13].

\section{What are the advantages/limitations of intra-venous thrombolysis alone?}

The main limitations of IV rTPA include its inability to provide any diagnostic information, a short time window for use, inadequate recanalization rates, and poor specificity for the site of arterial occlusion. The advantages of IV rTPA remain its relative ease of administration, widespread availability, and proven efficacy within 3/4.5 h of AIS.

In angiographically based studies, recanalization of the ICA and MCA was rare after intravenous thrombolysis [14-16]. Recent studies that evaluated the response to intravenous rTPA using transcranial Doppler or CT angiography suggest that only $13 \%$ to $18 \%$ of patients with proximal MCA occlusions recanalize after administration of intravenous rTPA $[17,18]$. Lee et al. demonstrated recanalization of major vessel occlusions only in $23 \%$ of patients [17]. If recanalization is taken as an important prerequisite for better stroke outcome, IV thrombolysis for major vessel occlusions such as MCA, ICA or basilar arteries is not justified as the optimal strategy. Although recanalization does not automatically translate into good stroke outcome and other factors such as time of intervention, collateral supply, degree of stroke severity, and comorbid conditions play a major role, prompt reperfusion retains its paramount importance. While the only imaging required before giving rTPA is a noncontrast head CT, to remain time efficient, it leaves much to be desired in terms of diagnostic accuracy of clot location, ischemic penumbra, collateral blood flow, and potential time window for efficacy of treatment. Therefore, although expediency of treatment is paramount for improving outcome, in patients with occlusion of the internal carotid artery (ICA) or M1 segment of the MCA, systemic thrombolysis may not be capable of achieving vessel recanalization due to the large clot burden. Can we do better with IA thrombolysis or combined IA/IV thrombolysis or combined $\mathrm{IV} /$ mechanical and other endovascular thrombolysis in these patients with large vessel occlusion, even if they present within $3 \mathrm{~h}$ of stroke onset? This precludes the FDA approval and the stroke guidelines. The argument in favor is given below.

\section{Intra-arterial thrombolysis}

Recent advances in the field of neurointerventional radiology, with the development of extremely soft, compliant microcatheters and steerable microguide wires, along with high contrast agents, have made it feasible and safe to access the major intracranial blood vessels around the Circle of Willis from a percutaneous transfemoral approach under local anaesthesia. Intra-arterial thrombolysis has been used most successfully in patients with acute MCA occlusion. Trials have used IA thrombolysis to at least $6 \mathrm{~h}$ from stroke onset in patients with MCA occlusion. Other potential candidates for IA thrombolysis include patients with extracranial carotid artery occlusion, intracranial carotid artery $\mathrm{T}$ occlusion and basilar artery occlusion.

Thrombolytic agents used for IA thrombolysis have been rTPA, urokinase (UK), pro-urokinase (scu-PA), reteplase, desmoteplase, tenecteplase (TNK), streptokinase, staphylokinase, plasmin, and microplasmin [19-41].

Compared to IV therapy, localized IA thrombolysis has the theoretical advantage of achieving more complete recanalization with less fibrinolytic drug, and clot lysis can be assessed with follow-up angiograms. Drug infusion can be stopped when clot lysis is achieved, leading to potentially less thrombolytic drug being used. Finally, with IA thrombolysis, treatment has been initiated up to $6 \mathrm{~h}$ after symptom onset. This generally expands the time window, which increases the number of patients who potentially can benefit from thrombolysis beyond the time limit of $3 \mathrm{~h}$ or perhaps $4.5 \mathrm{~h}$ (as is evidenced from the European Co-operative Acute Stroke Study - ECASS III trial results [13]).

Two randomized trials comparing IA recombinant pro-urokinase (r-pro-UK) plus IV heparin vs. IV heparin have been conducted in patients with occlusion of the MCA (M1 and M2) of $<6$ h' duration. In the Prolyse in Acute Cerebral Thromboembolism (PROACT) I trial [19], 40 patients with MCA occlusion were treated with either IA r-pro-UK $(n=26)$ or placebo $(n=14)$. All patients received IV heparin. The protocol initially specified 
a heparin dose of a $100 \mathrm{IU} / \mathrm{kg}$ bolus and an infusion of $1000 \mathrm{U} / \mathrm{h}$ for $4 \mathrm{~h}$. After 16 patients were randomized, the heparin dose was reduced to a $2000 \mathrm{IU}$ bolus and an infusion of $500 \mathrm{IU} / \mathrm{h}$ for $4 \mathrm{~h}$ on the recommendations of the safety committee. The study drug was started on average $5.5 \mathrm{~h}$ after the onset of symptoms. Recanalization rates were significantly higher with r-pro-UK (58\%) than with placebo (14\%; 2 -sided $p=0.017)$. There was no significant difference in the rate of early symptomatic hemorrhagic transformation, which occurred in $15.4 \%$ of the r-pro-UK patients and $7.1 \%$ of the placebo-treated patients $(2 p=0.64)$. Ninetyday mortality rates ( $4 \%$ in the pro-UK group vs. $7 \%$ in the control group) and good clinical outcomes (30.4\% vs. $21.4 \%$ ) favored treatment with r-pro-UK but did not reach statistical significance [19]. Recanalization rates and the risk of brain hemorrhage were influenced by the dose of heparin [19].

The PROACT II study [20] was designed to further test the efficacy and safety of IA r-pro-UK in patients with MCA occlusion of $<6 \mathrm{~h}$ duration. More than 1200 patients were evaluated for inclusion in the trial and 474 patients underwent conventional cerebral angiogram screening. One hundred eighty patients had angiogram-confirmed MCA occlusions and were randomized to receive $9 \mathrm{mg}$ of IA r-proUK plus heparin $(n=121)$ or heparin alone $(n=59)$. The heparin dose was the same in both groups (2000 $\mathrm{U}$ bolus and a $500 \mathrm{U} / \mathrm{h}$ infusion of heparin for $4 \mathrm{~h}$ ). A clinically and statistically significant benefit favored r-pro-UK in the primary outcome analysis, with $40 \%$ of the treated patients recovering to an $\mathrm{mRS}$ of $\leq 2$ compared with $25 \%$ of control patients (ARR 15\%, $p=0.043$, relative risk reduction $60 \%$ ). Mortality was $25 \%$ in the r-pro-UK group and $27 \%$ in the control group. Symptomatic intracranial hemorrhage (ICH) occurred in 10\% of the r-pro-UK group and $2 \%$ of IN controls ( $p=0.063)$. The recanalization rate (thrombolysis in myocardial infarction 2 or 3 ) was $66 \%$ for those on r-pro-UK vs. $18 \%$ for the controls ( $p<0.001$ ). Patients recruited in PROACT II had moderate-tosevere strokes with a median baseline NIHSS of 17. The median time to start IA treatment was $5.3 \mathrm{~h}$. The benefits of $r$-pro-UK were maximal in patients with baseline NIHSS scores of 11-20 [20].

The Middle Cerebral Artery Embolism Local Fibrinolytic Intervention Trial (MELT) randomized 114 patients in Japan with MCA stroke within $6 \mathrm{~h}$ to IA UK or standard therapy [42]. No intravenous thrombolysis was allowed in either group. Adjunctive mechanical disruption of clot was allowed in the UK group. The study was stopped prematurely once IV thrombolysis was approved in Japan. The primary endpoint of favorable outcome (mRS 0-2) at 90 days occurred more often in the IA group $(49.1 \%$ vs. $38.6 \%$, OR $1.54,95 \% \mathrm{Cl} 0.73$ to
3.23, $p=0.345)$, but was not statistically significant. The preplanned secondary endpoint of excellent outcome (mRS 0-1) at 90 days was significantly higher in the UK group (42.1\% vs. $22.8 \%$, OR 2.46 , $95 \% \mathrm{Cl} 1.09$ to $5.54, p=0.045)$. It should be noted that the MELT trial randomized patients with a lower baseline stroke severity compared to the PROACT II trial (NIHSS 14 vs. 17) and treated patients earlier; this may partially explain the lack of statistical significance for the primary endpoint (mRS 0-2 at 90 days) in the MELT trial. Furthermore, the MELT study may have been underpowered to detect a difference between groups due to the premature termination of the study once IV thrombolysis became available in Japan [43].

Ducrocq et al. conducted a small randomized multicenter trial comparing IV UK vs. IA UK given within the first $6 \mathrm{~h}$ of AIS [44]. Patients received $900,000 \mathrm{U}$ of UK via IV $(n=14)$ or IA $(n=13)$ routes. Due to safety concerns the study was stopped early. A total of 7 patients (26\%) died: 4 in the IV group and 3 in the IA group. Although the authors reported a greater and earlier improvement in the IA group, there was no difference in the main outcomes studied.

It is unclear whether IA thrombolysis or IV thrombolysis is more effective for ischemic stroke with hyperdense middle cerebral artery sign (HMCAS) on computed tomography (CT). Mattle et al. [45] compared IA thrombolysis and IV thrombolysis in stroke patients with HMCAS. The comparison of data was from 2 stroke units with similar management of stroke associated with HMCAS, except that 1 unit performed IAT with urokinase and the other IVT with plasminogen activator. Time to treatment was up to $6 \mathrm{~h}$ for IA thrombolysis and up to $3 \mathrm{~h}$ for IV thrombolysis. Outcome was measured by mortality and the modified Rankin Scale (mRS), dichotomized at 3 months into favorable (mRS 0 to 2) and unfavorable (mRS 3 to 6). One hundred twelve patients exhibited a HMCAS, of whom 55 were treated with IA thrombolysis and 57 with IV thrombolysis. Stroke severity at baseline and patient age were similar in both groups. Mean time to treatment was longer in the IA thrombolysis group (244 $\pm 63 \mathrm{~min}$ ) than in the IV thrombolysis group (156 $\pm 21 \mathrm{~min}, p=0.0001$ ). However, favorable outcome was more frequent after IA thrombolysis $(n=29,53 \%)$ than after IV thrombolysis $(n=13$, $23 \%, p=0.001)$, and mortality was lower after IA thrombolysis $(n=4,7 \%)$ than after IV thrombolysis ( $n=13,23 \%, p=0.022$ ). After multiple regression analysis IA thrombolysis was associated with a more favorable outcome than IV thrombolysis ( $p=0.003)$ but with similar mortality $(p=0.192)$. In this observational study IA thrombolysis was more beneficial than IV thrombolysis in the specific group of stroke patients presenting with HMCAS on CT, 
even though IA thrombolysis was started later. Their results indicate that a randomized trial comparing both thrombolytic treatments in patients with middle cerebral artery occlusion is warranted [45].

Quershi et al. [46], however, found favorable results even in patients with HMCAS thrombolyzed intravenously within $3 \mathrm{~h}$ of stroke onset. Class $1 \mathrm{~b}$ angiographic proof for reperfusion and good outcomes for large vessel came from the PROACT II trial [20].

Despite the findings of PROACT II the FDA did not approve proUK for use. Because proUK is not commercially available, most interventional neuroradiologists use UK or rTPA when performing IA thrombolysis. No randomized controlled trials of IA thrombolysis with rTPA for acute stroke have been performed. The results of IA thrombolysis using t-PA or UK in selected patients have generally been favorable, or clinical outcomes have been better than anticipated, despite increases in the rate of symptomatic ICH. Several retrospective studies have suggested that IA thrombolysis is superior to IV thrombolysis in patients with ICA, BA or M1 occlusions associated with the dense MCA sign. A few smaller studies of ICA or BA occlusion suggest the superiority of IA thrombolysis over IV thrombolysis. Furthermore, even though data on reopening major arterial occlusion after AIS with devices is mainly registry based, the results are comparable to those reported in the PROACT study without a significant increase in symptomatic hemorrhage.

\section{Intra-arterial thrombolysis in the vertebrobasilar territory}

The natural history of basilar artery occlusion is extremely poor, with mortality ranging from $83 \%$ to $91 \%$ [47]. Basilar artery occlusion is usually due to atherothrombosis. There is a high incidence of residual stenosis after basilar artery recanalization, which often requires adjuvant therapies including angioplasty, antithrombotic and antiplatelet agents, and stenting. Good clinical outcomes are strongly associated with recanalization after thrombolytic therapy. Hacke et al. described 65 consecutive patients with vertebrobasilar occlusions treated either with local IA UK or IA streptokinase (SK) plus heparin $(n=43)$ or conventional antiplatelet/ anticoagulation therapy $(n=22)$ [48]. The recanalization rate in thrombolysis patients was $44 \%$ (19 of 43 ). All patients without recanalization died whereas 14 of 19 patients with recanalization survived, and 10 had a favorable outcome. The mortality rate with conventional therapy was $86 \%$ compared to $67 \%$ with thrombolysis. The rate of brain hemorrhage with clinical deterioration was $7 \%$ in patients who underwent thrombolysis [48].

Recanalization rates depend on the location of the vertebrobasilar occlusion (VBO) [48-50]. Distal basilar occlusions have higher recanalization rates than proximal ones. The timing of IA vertebrobasilar thrombolysis is often a difficult decision. The presence of coma or tetraparesis for several hours portends a poor prognosis despite recanalization. Such symptoms however may not preclude survival, and recovery has been documented after successful recanalization in such patients.

The time window for thrombolysis may be longer in VBO, with many series reporting time-window durations of up to $24 \mathrm{~h}, 48 \mathrm{~h}$ and even $72 \mathrm{~h}$ after symptom onset in patients with stuttering courses. Some studies suggested good outcomes in patients receiving even delayed treatment. A longer time window may be due to a higher ischaemic tolerance or improved collateralization in the posterior circulation [47]. Cross et al. [51], reporting on 20 patients with basilar artery thrombosis who received IA thrombolysis, found that better collateral blood flow was correlated with improved responses to thrombolysis and with longer tolerance of ischaemia. Patients with proximal basilar artery thrombosis did not seem to have the same benefit. Patients with vertebrobasilar ischaemia often have chronic atherosclerotic disease which allows collaterals to develop over time.

There may be two distinct populations of patients with VBO as hypothesized by Cross [51]. Patients with a progressive stuttering course may have improved collateral circulation and better outcomes despite later treatment than patients with sudden onset of severe deficits owing to poor collaterals, even though they may be brought to treatment earlier. Superselective IA angiography and local IA administration of thrombolytic agents is now considered most optimal for more complete recanalization of acute basilar thrombus. Complete reperfusion of the posterior circulation is taken as a conditional sine qua non for the most favorable stroke outcome after basilar artery occlusion. There are arguments raised that although optimal, this procedure requires expertise and adequate infrastructure which makes it amenable only to a few selected centers.

\section{Combined intra-venous and intra-arterial thrombolysis}

One major disadvantage of IA thrombolysis is the delay to treatment because of the waiting period for initiation and performance of cerebral angiography. The Emergency Management of Stroke (EMS) Study [52] and the Interventional Management of Stroke (IMS) Trial [53] combined IV and IA thrombolysis. This approach combines the advantages of 2 treatment strategies. IV therapy is initiated without delay but at a lower dose, which allows some of the tPA to be given intra-arterially for the higher recanalization rates that potentially 
can be achieved with IA treatment. The EMS or the Bridging Trial randomized 35 patients to either combination IV/IA thrombolysis $(n=17)$ or placebo/IA thrombolysis $(n=18)$ within $3 \mathrm{~h}$ of onset of symptoms. Complete recanalization rates were better in the combination IV/IA group (6/11) vs. the placebo/IA group (1/10) ( $p=0.03)$; however, neurological outcomes at 90 days did not differ between groups. The mortality rate trend was higher in the combination (IV/IA) group (29\%) vs. the placebo/IA group (5.5\%) $(p=0.06)$. The small sample size of this study precluded any definitive conclusions [48]. The EMS trial demonstrated that the combined approach is feasible, reasonably safe, and worthy of further study [52]. Although the Interventional Management Study (IMS II) [53], a trial that combined IV and IA therapy, was considered a negative trial in terms of the primary outcome, the group treated with combined IV and IA therapy had a better outcome than the placebo treated patients of the NINDS trial and if secondary outcome measures were considered (Barthel Index and the Global Scale), a statistically better outcome was seen with combined therapy compared to the IV treated group of the NINDS trial. Of the 74 patients who underwent an angiogram after receiving IV rt-PA ( $0.6 \mathrm{mg} / \mathrm{kg}$ with a $15 \%$ bolus), the majority achieved a TIMI grade 2-3 flow after additional IA thrombolysis (43\% treated within $3 \mathrm{~h}$ and $13 \%$ between $3-4 \mathrm{~h}$ ). Of the 28 patients with ICA occlusion, $56 \%$ achieved a TIMI reflow of $2-3$, which correlated with the outcome of a $\mathrm{mRS} \leq 1$. Of the remaining patients with ICA occlusions who had a TIMI of $0-1$, only $12 \%$ had a similar good outcome. Two important conclusions can be drawn from the IMS II trial. Firstly, recanalization was achieved only after rescue IA therapy in the majority of patients. Secondly, those patients treated with the combined therapy within $3 \mathrm{~h}$ had a significantly better outcome than those treated within the 3-4 $\mathrm{h}$ window. Whether we can do better with the combined or operator-based selected device and/or thrombolysis remains a question that will hopefully be answered by the 2 ongoing trials, Interventional Management Study 3 (IMS 3) and MR RESCUE.

\section{What are the limitations of intra-arterial thrombolysis?}

The main disadvantages of IA thrombolysis are the logistic factors involved such as the need to assemble the angiography team and confirm occlusion angiographically before administration of thrombolysis. Once IA therapy commences, the average time to recanalization ranges from 1.9-2.8 $\mathrm{h}$ [46]. One approach to minimize the delay in thrombolysis using IA therapy is to use a combination of a reduced dose of IV rTPA $(0.6 \mathrm{mg} / \mathrm{kg})$ within $3 \mathrm{~h}$ and then continue therapy using
IA thrombolysis in patients who do not respond to IV thrombolysis. The other potential disadvantage of IA thrombolysis is the invasiveness of angiography and the expertise required. It will be unlikely that routine IA thrombolysis will be feasible in most practice settings. Although the risk of serious complications is relatively low, in a large retrospective analysis of about 20,000 patients who underwent cerebral angiography at the Mayo clinic, stroke and death occurred in $0.15 \%$ and $0.06 \%$ of patients, respectively [54]. Other complications include access-site hematoma (4.2\%); nausea, vomiting or transient hypotension (1.2\%); anaphylaxis $(0.03 \%)$; and acute renal failure $(0.04 \%)$. Stroke occurred more frequently in patients with underlying atherosclerotic disease (0.25\%) [54]. The relative costs of IA vs. IV thrombolysis have not been reported but it is logical to assume that the direct cost of treatment with IA thrombolysis would be higher. However, when the long-term implications of stroke mortality and morbidity are considered, these cost implications may be better for IA thrombolysis.

\section{Newer thrombolytic agents}

One of the 2 major problems in crossing the 3-h time window is the propensity for $\mathrm{ICH}$. Several second- and third-generation thrombolytic agents (tenecteplase, reteplase, desmoteplase, plasmin, microplasmin) are being tested. The goal is to create new thrombolytic agents with longer half-lives that permit bolus administration, and have better rates of restoration of flow and reduced rates of $\mathrm{ICH}$.

Desmoteplase: The recently completed phase II trial Desmoteplase in Acute Stroke study (DIAS) of an rt-PA molecule derived from bat saliva used diffusion/perfusion MRI to enroll patients and assess the biological activity of different doses [27]. Patients were included in the study between 3 and $9 \mathrm{~h}$ after stroke onset, if they demonstrated a perfusion lesion volume $\geq 20 \%$ above the baseline diffusion lesion volume. Magnetic resonance angiography (MRA) was also performed and all $3 \mathrm{MRI}$ modalities were repeated several hours after completion of thrombolytic therapy to evaluate the efficacy of reperfusion. Lower weight-adjusted dosing demonstrated a reasonable safety profile and dramatic reperfusion efficacy. Clinical outcomes tended to be more favorable in treated patients as well. This pilot study is important because it confirms the safety of weight-adjusted desmoteplase in ischaemic stroke patients when administered up to $9 \mathrm{~h}$ after stroke onset, as well as the intended biological effect of inducing reperfusion [26, 27].

Tenecteplase: TNK-tPA is a more fibrin-specific variant of t-PA. It also has a longer half-life and increased resistance to plasminogen activator 
inhibitor type 1 (PAI-1) which allows for dosing as a single bolus. Pilot studies are under way to test its efficacy [24].

\section{Combination of adjunctive therapy with thrombolysis}

Glycoprotein (Gp) IIb/IIla inhibitors block the final common pathway of platelet aggregation, and have demonstrated efficacy in preventing thrombus formation in acute coronary syndromes and percutaneous coronary interventions (PCIs). In acute ischemic stroke, a dose-escalation study demonstrated that the Gp IIb/IIla inhibitor abciximab is safe when given as a $0.25 \mathrm{mg} / \mathrm{kg}$ bolus and $0.125 \mathrm{mg} / \mathrm{kg} / \mathrm{min}$ infusion. The Emergent Stroke Treatment Trial (ABESTT) study [55] suggests a possible efficacy of abciximab in acute stroke with a longer therapeutic window and a better safety profile than rt-PA given within $3 \mathrm{~h}$. A phase II trial is under way in AIS of $<6 \mathrm{~h}$ duration. Some centers use Gp IIb/IIla inhibitors as adjunctive therapy with IA thrombolysis in AIS [35]. There are also reports of acute intervention with angioplasty and stenting. Some trials are underway using ultrasound waves as an adjunct to better lyse the clot when using IV or IA thrombolysis. The Neuroflo Catheter is an endovascular device that is in clinical study. It uses inflatable balloons that are placed in the abdominal aorta to augment cerebral blood flow without increasing blood pressure; randomized controlled trials are underway to assess the utility of this therapy. Several catheters that control body temperature and induce hypothermia for the treatment of stroke are also currently under investigation. Data are not adequate to comment further on their safety or efficacy.

\section{Mechanical clot lysis}

Mechanical approaches to ischemic stroke are attractive because they may obviate the need for thrombolytic drugs which increase the risk of reperfusion hemorrhage. The AngioJet catheter has been used to fragment and suck in the clot. It uses a high-velocity stream of saline directed back into the catheter to create a localized low-pressure zone at the distal tip. The thrombus is trapped, broken into small particles and evacuated within minutes via the Bernoulli effect. The system is currently used in saphenous vein graft thrombectomy but has also been modified for neurovascular use. Distal embolization of particles is of potential concern. Other means of delivering energy to fragment the clot are being developed, including use of ultrasound and laser devices. The current limitation of these mechanical devices is their relatively large catheter size and less flexibility, which limit access to the tortuous vessels of the intracranial circulation. The Mechanical Embolus Removal in Cerebral Ischemia (MERCI) Trial evaluated the safety of the MERCI Retriever System [56]. MERCI trial was a nonrandomized study using historical controls from PROACT II [20]. This study included patients with an occlusion of a major vessel who could be treated within $8 \mathrm{~h}$ of symptom onset. The primary goal of the $\mathrm{MERCl}$ study was to achieve a recanalization rate greater than seen in the control arm of PROACT II (18\%). A total of 151 rTPAineligible patients were enrolled in the MERCI trial, and the device was deployed in 141 of these patients; recanalization occurred in $48 \%$ of treated patients, a percentage that is significantly greater than the spontaneous recanalization rate seen in PROACT II $(p<0.0001)$. The neurologic outcome was a secondary outcome measure in this study and was assessed by comparing the outcome of patients undergoing mechanical thrombectomy with that of patients in the control arm of PROACT II. In comparison to PROACT II, mortality was not decreased in the $\mathrm{MERCl}$ trial, although $\mathrm{MERCl}$ patients appeared to have more severe strokes (Table II - compares data from PROACT II, MERCI, IMS I, IMS II with patients in the NINDS study who have an NIHSS of 10 or more [57]). In fact, mortality appeared to be increased (54.2\%) in patients who could not be recanalized, suggesting that the procedure may harm this population.

The Multi MERCI trial was the follow up to $M E R C l$. This trial included patients who received intravenous rTPA within $3 \mathrm{~h}$ time window but had persistent vessel occlusion. Patients who were rTPAineligible were also included [58, 59]. Patients had to be treated within $8 \mathrm{~h}$ of stroke onset. The main finding of this study supports the safety for mechanical thrombectomy in patients who had already received intravenous rTPA according to the FDA-approved indications. The study results suggest that "rescue therapy" with the MERCI Retriever may be considered for patients who fail to recanalize after intravenous thrombolytic therapy. MERCI patients had more severe stroke (median NIHSS =19) and comprised a more heterogeneous stroke population than did the patients in either PROACT II or the NINDS rTPA trial, which limits the validity of the comparison of these trials. However, based on the design and findings of the $\mathrm{MERCl}$ trial, it appears that the device is capable of recanalizing blood vessels in patients with stroke. These trials however do not provide definitive evidence that treatment with the Merci Retriever improves neurologic outcome.

A few other smaller studies such as EKOS, TRUMBI and CLOT BUST [60] evaluated the additional benefits of using thrombolytics in combination with exposure to ultrasound. Theoretically, this approach would increase the 
Table II. Comparison of endovascular trials and patients from the NINDS trial with a baseline NIHSSS $\geq 10$

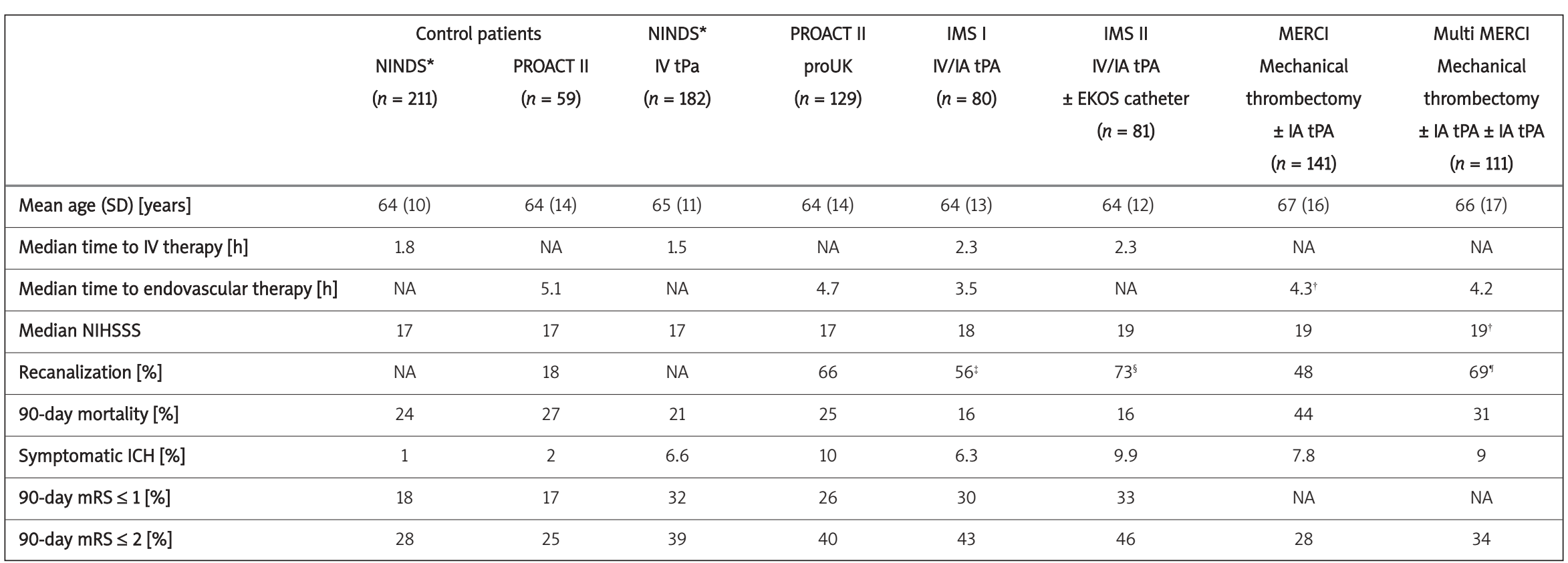

*These data only include patients with NIHSSS $\geq 10$

Mean (median not report)

*Recanalization rate among patients undergoing an endovascular procedure

IIn patients treated with the EKOS catheter as well as IA TPA

In patients treated with mechanical thrombectomy plus adjuvant therapy

IA - intra-arterial, ICH - intracerebral hemorrhage, IMS - Interventional Management of Stroke, IV - intravenous, MERCI - Mechanical Embolus Removal in Cerebral Ischemia, mRS - modified Rankin score, NA - not available or not applicable, NIHSSS - National Institutes of Health Stroke Scale Score, NINDS - National Institute of Neurological Disorders and Stroke, PROACT - Pro-Urokinase for Acute Cerebral Thromboembolism, proUK - pro-urokinase, SD - standard

deviation, tPA - tissue plasminogen activator 
accessibility of the drug to the clot. Using the same protocol as IMS I, the IMS II study evaluated a new approach to recanalization using the novel endovascular device known as the EKOS Neuro Wave Catheter [53], along with IA rTPA infusion. Among patients treated with IA rTPA and the EKOS Neuro Wave Catheter, $73 \%$ of the vessels were recanalized at the end of the procedure (compared with $56 \%$ in the IMS I). Based on these data, an NIH-funded phase III trial is underway to compare intravenous thrombolysis with a combined approach using a modified dose/ administration protocol for intravenous rTPA followed by an attempt at recanalization using IA thrombolysis and other endovascular techniques, including mechanical thrombectomy (with the Merci Retriever) and the EKOS Neuro Wave Catheter.

The safety and performance of the Penumbra System (PS), a novel mechanical device designed to reduce clot burden in acute stroke due to largevessel occlusive disease was tested in a prospective, single arm, independently monitored and core laboratory adjudicated trial which enrolled subjects with acute ischemic stroke, presenting within $8 \mathrm{~h}$ of symptom onset and an angiographically verified occlusion (Thrombolysis in Myocardial Infarction [TIMI] grade 0 or 1 ) of a treatable intracranial vessel [61]. The primary end point was revascularization of the target vessel to TIMI grade 2 or 3 . Secondary end points were the proportion of subjects who achieved a mRS score of 2 or less or a 4-point improvement on the NIHSS score at 30-day followup, as well as all-cause mortality. Of the 23 patients enrolled, and 21 target vessels treated in 20 subjects by the PS, (mean age $=60$ years, mean $\mathrm{mRS}$ score $=4.6$, and mean NIHSS score $=21$ ), all 21 of the treated vessels (100\%) were successfully revascularized by the PS to TIMI 2 or 3. At 30-day follow-up, 9 subjects (45\%) had a 4-point or more NIHSS improvement or an mRS of 2 or less. The allcause mortality rate was $45 \%$ (9 of 20), which is lower than expected in this severe stroke cohort, where $70 \%$ of the subjects at baseline had either an NIHSS score of more than 20 or a basilar occlusion [61]. Following this study PS has also been approved by the FDA for clinical use.

\section{Reperfusion strategies in stroke subtypes}

Use of aggressive interventional strategies is often advocated for basilar artery thrombosis, considering the devastating mortality and morbidity associated with it. However, intervention in patients who have been quadriplegic or deeply comatose for more than $3 \mathrm{~h}$ does not portend a good recovery [62]. The Basilar Artery Interventional Cooperation Study is a prospective, nonrandomized study that will compare outcomes among patients who receive antithrombotics, intravenous thrombolytics, and endovascular therapy.

Patients with ICA occlusion rarely experience good recanalization with IV thrombolysis [12, 63, 64]. In MERCI and Multi MERCI trials, out of the 80 patients with carotid occlusion, 53\% were recanalized with mechanical thrombectomy alone and $63 \%$ were recanalized with mechanical thrombectomy and adjunctive endovascular therapy [65]. Good clinical outcome (modified Rankin Score $[\mathrm{mRS}] \leq 2$ ) was achieved in 39\% with recanalization and in 3\% without recanalization emphasizing the concept that recanalization may not the sole but an inherently important component for achieving neurological recovery [65]. Mortality was $30 \%$ in the recanalized group and $73 \%$ in the non-recanalized group [65].

\section{Expanding the therapeutic time window}

After vascular occlusion, there is a heterogeneous depression of cerebral blood flow (CBF) in the territory of the occluded artery. The penumbra is identified as the brain region receiving regional CBF ( $r C B F)$ between two critical values. The first, higher critical value, is associated with neuronal paralysis: brain areas receiving rCBF less than $18-20 \mathrm{ml} / 100 \mathrm{~g} / \mathrm{min}$ do not function. The second, lower critical value, is associated with cell death; brain areas receiving less than $8-10 \mathrm{in} \mathrm{ml} / 100 \mathrm{~g} / \mathrm{min}$ do not survive, and this area becomes the core of the infarction [1, 2]. Neurons in the penumbra are sometimes identified as idling to suggest that they are salvageable, although the mechanism of such a phenomenon is unknown. Cell death in the core is rapid whereas cells in the penumbra may survive up to several hours.

A substantial amount of information is now available from positron emission tomography (PET) and MRI studies, which show that in some patients potentially salvageable ischemic tissue exists for many hours after stroke onset [66-84]. The availability of PET is limited and the technique is time-consuming, and hence it is likely to remain an imaging tool restricted to research centers. Diffusion-perfusion MRI and CT perfusion studies are now widely available at many clinical facilities. The PWI-DWI mismatch on MRI provides a rapid imaging marker of potentially salvageable ischemic tissue which can be widely used to identify potentially treatable ischemic stroke patients irrespective of the time from onset. Hopefully, using this approach, patient selection will be based on a pathophysiological assessment rather than rigid time window periods. This should expand the therapeutic time window for both thrombolysis and neuroprotection.

In future, absolute value maps of perfusion and diffusion are likely to be generated, and estimations 
provided as to what percentage of ischemic tissue is highly likely to be irreversibly injured, what percentage is not at risk for infarction, and what percentage is at risk for infarction but can be salvaged with appropriate treatment. The availability of such a 3-compartment assessment of ischemic tissue will move acute stroke treatment into a new era of time-independent decisionmaking.

DWI may delineate infarcted brain tissue within minutes, although there is growing evidence that in the very early stage of stroke there may be reversible DWI changes in up to $45 \%$ of patients experiencing recanalization after treatment with recombinant tissue plasminogen activator (rt-PA). However, in most instances, these lesion reversals are only minor or partial and frequently not permanent. There are contradictory data as to whether a DWI/apparent diffusion coefficient threshold for irreversible ischaemia exists. PWI defines the area of cerebral hypoperfusion. The absolute volume difference or ratio of PWI to DWI reveals the ischemic tissue at risk of irreversible infarction. The presence of vessel occlusion on magnetic resonance angiography (MRA) is associated with a PWI/DWI mismatch. Therefore, stroke MRI defines the ideal candidate for thrombolysis. In addition, early recanalization achieved by thrombolysis results in significantly smaller infarcts and a better clinical outcome. The concept of diffusion/perfusion mismatch has received the most attention and is best demonstrated by the Diffusion and Perfusion Imaging Evaluation for Understanding Stroke Evolution (DEFUSE) study [83]. DEFUSE showed that patients with a perfusion abnormality that was at least $20 \%$ of their diffusion abnormality on initial MRI seemed to benefit from delayed (3-6 h) thrombolysis/recanalization compared with patients who did not recanalize and those who did not have a diffusion/perfusion mismatch. The concept of diffusion/perfusion mismatch as a target for recanalization is currently being tested in a prospective, randomized, controlled trial known as MR RESCUE. In this study, patients are randomized within $8 \mathrm{~h}$ onset to either endovascular recanalization with a $\mathrm{MERCI}$ Retriever or standard care. Randomization is stratified by the initial magnetic resonance diffusion/perfusion pattern.

A comparison of computed tomography/computed tomography angiography/CTA source image analysis (CT/CTA/CTA-SI) findings with MRI (DWI) and MRA reveals equal accuracy of CTA and MRA, close to equal accuracy of infarct volumes according to CTA-SI compared with DWI, and a predictive value of poor collaterals for infarct growth. Therefore, CT/CTA/CTA-S1 may provide information similar to that of the PWI/DWI mismatch concept.
There is growing evidence for emerging strategies that have the potential to extend cerebral reperfusion therapy beyond the 3-h/4.5-h time window. Approaches to this include: (i) intra-arterial (IA) pharmacological reperfusion approaches, combined intravenous-intra-arterial (IV/IA) fibrinolysis and combined fibrinolytics and glycoprotein (Gp)Ilb/IIla agents; (ii) emerging endovascular mechanical reperfusion strategies including IA thrombectomy (clot retrieval devices and suction thrombectomy devices), mechanical disruption (micro-guidewire passage, laser photo-acoustic emulsification, primary intracranial angioplasty); (iii) augmented fibrinolysis by endovascular ultrasound; (iv) multimodal imaging with MRI or CT to rapidly assess the infarct core, penumbra, site of vessel occlusion and hemorrhagic propensity of the tissue, enabling improved selection of patients for reperfusion therapy beyond any arbitrary fixed time window; (v) newer thrombolytic agents; (vi) adjunctive therapies such as neuroprotectants.

Given all the presently available data, if we follow the guidelines of intravenous thrombolysis within the first $3 \mathrm{~h} / 4.5 \mathrm{~h}$ after stroke onset for all AIS, we have a less than $20 \%$ chance of reopening large arteries (ICA, MCAM1, or BA, especially proximal BA occlusions) with IV t-PA vs. a $60-70 \%$ chance with IA thrombolysis. While it is not entirely clear if reopening blood vessels equals reperfusion, all the present data from meta-analysis of thrombolytic trials, retrospective large database analysis and case series suggest that it does. Why else would we have resolution of perfusion deficits and shrinkage/stabilization hyperintensity on diffusion weighted imaging after successful establishment of reflow?

Maybe it is time to reassess our options when we consider the mode of thrombolysis for AIS presenting within the 3- and 4.5-h time window. The paradigm may be as follows. In centers with adequate infrastructure and know-how, in patients presenting with AIS: perform CT/CTA/perfusion CT or MRI/MRA/perfusion MRI. If patients have a major large vessel occlusion (ICA, M1 or BA) consider IA treatment. If significant delays in starting IA are expected, IV thrombolysis can be initiated followed by IA treatment if the patient fails to recanalize. For all other cases consider IV thrombolysis.

\section{References}

1. Astrup J, Symon L, Branston NM, Lassen NA. Cortical evoked potential and extracellular $\mathrm{K}+$ and $\mathrm{H}+$ at critical levels of brain ischemia. Stroke 1977; 8: 51-7.

2. Heiss WD. Progress in cerebrovascular disease: flow thresholds of functional and morphological damage of brain-tissue. Stroke 1983; 14: 329-31.

3. Hacke W, Del Zoppo GJ, Harker LA. Thrombosis and cerebrovascular disease. In: Recent advances in diagnosis 
and management of stroke. Poeck K, Ringelstein EB, Tacke W (eds). Springer-Verlag, USA 1987; 59-64.

4. Fieschi C, Argentino C, Lenzi Gl, et al. Clinical and instrumental evaluation of patients with ischemic stroke within the first six hours. J Neurol Sci 1989; 91: 311-21.

5. The National Institute of Neurological Disorders and stroke rt-PA stroke study group. Tissue plasminogen activator for acute ischemic stroke. N Engl J Med 1995; 333: 1581-7.

6. Hoffman JR. Tissue plasminogen activator for acute ischemic stroke: is the CAEP position statement too negative? Can J Emerg Med CJEM 2001; 3.

7. Mann J. Truths about the NINDS study: setting the record straight. West J Med 2002; 176: 192-4.

8. Adams HP Jr, Davis PH, Leira EC, et al. Baseline NIH Stroke Scale score strongly predicts outcome after stroke: a report of the Trial of Org 10172 in Acute Stroke Treatment (TOAST). Neurology 1999; 53: 126-31.

9. Wolpert SM, Bruckmann H, Greenlee $\mathrm{R}$, et. al. Neuroradiologic evaluation of patients with acute stroke treated with recombinant tissue plasminogen activator. The rTPA Acute Stroke Study Group. AJNR Am J Neuroradiol 1993; 14: 3-13.

10. Mori E, Yoneda $\mathrm{Y}$, Tabuchi $\mathrm{M}$, et al. Intravenous recombinant tissue plasminogen activator in acute carotid artery territory stroke. Neurology 1992; 42: 976-82.

11. del Zoppo GM, Poeck K, Pessin MS, et al. Recombinant tissue plasminogen activator in acute thrombotic and embolic stroke. Ann Neurol 1992; 32: 78-86.

12. Lee KY, Han SW, Kim SH, et al. Early recanalization after intravenous administration of recombinant tissue plasminogen activator as assessed by pre and postthrombolytic angiography in acute iscehmic stroke patients. Stroke 2007; 38: 192-3.

13. Alexondrov AV, Molina CA, Grotta JC, et al. Ultrasound enhanced systemic thrombolysis for acute ischemic stroke. N Engl J Med 2004; 351: 2170-8.

14. del Zoppo GJ, Higashida RT, Furlan AJ, Pessin MS, Rowley HA, Gent M. PROACT: a phase II randomized trial of recombinant pro-urokinase by direct arterial delivery in acute middle cerebral artery stroke. Stroke 1998; 29: 4-11.

15. Furlan A, Higashida R, Wechsler L, et al. Intra-arterial prourokinase for acute ischemic stroke: the PROACT II study: a randomized controlled trial: prolyse in acute cerebral thromboembolism. JAMA 1999; 282: 2003-11

16. Leker RR, Eichel R, Rafaeli G, Ben-Hur T. Novel therapies for acute ischemic stroke. Isr Med Assoc J 2006; 8: 788-92.

17. Qureshi Al, Ali Z, Suri MF, et al. Intra-arterial third generation recombinant tissue plasminogen activator (reteplaase) for acute ischemic stroke. Neurosurgery 2001; 49: 41-50.

18. Qureshi Al, Pande RU, Kim SH, Hanel RA, Kirmani JF, Yahia AM. Third generation thrombolytics for the treatment of ischemic stroke. Curr Opin Investig Drugs 2002; 3: 1729-32.

19. Lachak PA, Song D, Wei J, Zivin JA. Coadministration of NXY-059 and tenecteplase six hours following embolic strokes in rabbits improves clinical rating scores. Exp Neurol 2004; 188: 279-85.

20. Cornu C, Boutitie F, Candelise L, et al. Streptokinase in acute ischemic stroke: an individual patient data metaanalysis: The Thrombolysis in Acute Stroke Pooling Project. Stroke 2000; 31: 1555-60.

21. Furlan AJ, Eyding D, Albers GW, et al. Dose escalation of Desmoteplase for Acute Ischemic Stroke (DEDAS): Evidence of safety and efficacy 3 to 9 hours after stroke onset. Stroke 2006; 37: 1227-31.

22. Hacke W, Albers G, Al-Rawi Y, et al. The Desmoteplase in Acute Ischemic Stroke Trial (DIAS): a phase II MRI-based 19-hour window acute stroke thrombolysis trial with intravenous desmoteplase. Stroke 2005; 36: 66-73.

23. Collen D, Lijnen HR. Thrombolytic agents. Thromb Haemost 2005; 93: 627-30.

24. Sugg RM, Noser EA, Shaltoni HM, et al. Intra-arterial reteplase compared to urokinase for thrombolytic recanalization in acute ischemic stroke. AJNR Am J Neuroradiol 2006; 27: 769-73.

25. Arnold M, Schroth G, Nedeltchev K, et al. Intra-arterial thrombolysis in 100 patients with acute stroke due to middle cerebral artery occlusion. Stroke 2002; 33: 1828-33.

26. Brekenfeld C, Remonda L, Ndedltchev K, et al. Symptomatic intracranial hemorrhage after intra-arterial thrombolysis in acute ischemic stroke: assessment of 294 patients treated with urokinase. J Neurol Neurosurg Psychiatry 2007; 78: 280-5.

27. Ueda T, Sakaki S, Kumon Y, Ohta S. Multivariate analysis of predictive factors related to outcome at 6 months after intra-arterial thrombolysis for acute ischemic stroke. Stroke 1999; 30: 2360-5.

28. Pannel R, Gurewich V. Pro-urokinase: a study of its stability in plasma and of a mechanism for its selective fibrinolytic effect. Blood 1986; 67: 1215-23.

29. Furlan A, Higashida R, Weschsler L, et al. Intra-arterial prourokinase for acute ischemic stroke. The PROACT II study: a randomized controlled trial, prolyse in acute cerebral thromoblembolism. JAMA 1999; 282: 2003-11.

30. Zhang L, Zhang ZG, Zhang C, Zhang RL, Chopp M. Intravenous administration of a GPIIb/IIla receptor antagonist extends the therapeutic window of intraarterial tenecteplase-tissue plasminogen activator in a rat stroke model. Stroke 2004; 35: 2890-95.

31. Lopez-Atalaya JP, Roussel BD, Ali C, et al. Recombinant Desmodus rotundus salivary mechanism without exerting neurotoxic effects. Stroke 2007; 38: 1036-43.

32. Vanderschueren S, Van Vlaenderen I, Collen D. Intravenous thrombolysis with recombinant staphylokinase versus tissue-type plasminogen activator in a rabbit embolic stroke model. Stroke 1997; 28: 1783-8.

33. Ahmed N, Nasman P, Wahlgren NG. Effect of intravenous nimodipine on blood pressure and outcome after acute stroke. Stroke 2000; 31: 1250-5.

34. Fitt GJ, Farrar J, Baird AE, et al. Intra-arterial streptokinase in acute ischemic stroke: a pilot study. Med J Aust 1993; 159: 331-4.

35. Lapchak PA. Development of thrombolytic therapy for stroke: a perspective. Expert Opin Invest Drugs 2002; 11: 1623-32.

36. Lapchak PA, Araujo DM, Pakola S, Song D, Wei J, Zivin JA. Microplasmin: a novel thrombolytic that improves behavioral outcome after embolic strokes in rabbits. Stroke 2002; 33: 2279-84.

37. Hacke W, Kaste M, Bluhmki E, et al.; ECASS Investigators. Thrombolysis with alteplase 3 to 4.5 hours after acute ischemic stroke. NEJM 2008; 359: 1317-29.

38. Ogawa A, Mori E, Minematsu K, et al. Randomized trial of intra-arterial infusion of urokinase witin 6 hours of middle cerebral artery stroke: the middle cerebral artery embolism local fibrinolytic intervention trial (MELT) Japan. Stroke 2007; 38: 2633-9.

39. Saver JL. Intra-arterial fibrinolysis for acute ischemic stroke: the message of MELT. Stroke 2007; 38: 2627-8.

40. Ducrocq X, Bracard S, Tailandier L, et al. Comparison of intravenous and intra-arerial urokinase thrombolysis for acute ischemic stroke. J Neuroradiol 2005; 32: 26-32.

41. Mattle HP, Arnold M, Georgiadis D, et al. Comparison of intra-arterial and intra-venous thrombolysis for ischemic 
stroke with hyperdense middle cerebral artery sign. Stroke 2008; 39: 379-83.

42. Qureshi Al, Ezzeddine MA, Nasar A. Is IV tissue plasminogen activator beneficial in patients with hyperdense artery sign? Neurology 2006; 66: 1171-4.

43. Furlan AJ. Natural history of atherothromboembolic occlusion of cerebral arteries: carotid versus vertebrobasilar territories. In: Hacke W, del Zoppo GJ, Hirschberg $M$ (eds). Thrombolytic therapy in acute ischaemic stroke. New York: Spinger-Verlag 1991; 71-6.

44. Hacke W, Zeumer H, Ferbet A, Bruckmann H, del Zoppo GJ. Intra-arterial thrombolytic therapy improves outcome in patients with acute vertebrobasilar occlusive disease. Stroke 1988; 19: 1216-22.

45. Huemer M, Niederwieser V, Ladurner G. Thrombolytic treatment for acute occlusion of the basilar artery. J Neurol Neurosurg Psychiatry 1995; 58: 227-8.

46. Becker KJ, Monsein LH, Ulatowski J, Mirski M, Williams M, Hanley DF. Intra-arterial thrombolysis in vertebrobasilar occlusion. AJNR Am J Neuroradiol 1996; 17: 255-62.

47. Cross DT, Moran CIJ, Akins PT, Angtuaco EE, Diringer MN. Relationship between clot location and outcome after basilar artery thrombolysis. AJNR 1997; 18: 1221-28.

48. Lewandowski CA, Frankel M, Tomsick TA, et al. Combined intravenous and intra-arterial r-TPA versus intra-arterial therapy in acute ischemic stroke: Emergency Management of Stroke (EMS) Bridgiing Trial. Stroke 1999; 30 2598-605.

49. The Interventional Management of Stroke (IMS) II Study. Stroke 2007; 38: 2127-35.

50. Hacke W, Kaste M, Fieschi C, et al. Intravenous thrombolysis with recombinant tissue plasminogen activator for acute hemispheric stroke: The European Cooperative Acute Stroke Study (ECASS). JAMA 1995; 274: 1017-25.

51. Hacke W, Kaste M, Fieschi C, et al. Randomized doubleblind placebo-controlled trial of thrombolytic therapy with intravenous alteplase in acute ischemic stroke (ECASS II): Second European-Australasian Acute Stroke Study Investigators. Lancet 1998; 352: 1245-51.

52. Clark WM, Wissman S, Albers GW, Jhamandas JH, Madden KP, Hamilton S. Recombinant tissue-type plasminogen activator (Alteplase) for ischemic stroke 3 to 5 hours after symptom onset. The ATLANTIS Study: a randomized controlled trial, Alteplase Thromoblysis for Acute Noninterventional Therapy in Ischemic Stroke. JAMA 1999 282: 2019-26.

53. Hacke W, Donnan G, Fieschi C, et al. Association of outcome with early stroke treatment: pooled analysis of ATLANTIS, ECASS and NINDS rt-PA stroke trials. Lancet 2004; 363: 768-74

54. Kauffman TJ, Huston J $3^{\text {rd }}$, Mandrekar JN, Schleck CD, Thielen KR, Kallmes DF. Complications of diagnostic cerebral angiography: evaluation of 19,826 consecutive patients. Radiology 2007; 243: 812-9.

55. Abciximab Emergent Stroke Treatment Trial Investigators. Emergency administration of abciximab for treatment of patients with acute ischemic stroke: results of a randomized phase 2 trial. Stroke 2005; 36: 880-90.

56. Smith WS, Sung G, Starkman S, et al. Safety and efficacy of mechanical embolectomy in acute ischemic stroke results of the MERCI trial. Stroke 2005; 36: 1432-8.

57. Becker K. Endovascular treatment of acute stroke. Current treatment options in neurology 2007; 21: 70-6.

58. Smith WS. Safety of mechanical thrombectomy and intrravenous tissue plasminogen activator in acute ischemic stroke. Results of the multi Mechanical Embolus
Removal in Cerebral Ischemia (MERCI) trial, part I. AJNR Am J Neuroradiol 2006; 27: 1177-82.

59. Smith WS, Sung G, Saver J, et al. Mechanical thrombectomy for acute ischemic stroke: final results of the multi merci trial. Stroke 2008; 39: 1205-12.

60. Alexandrov AV, Wojner AW, Grotta JC; CLOTBUST Investigators. CLOTBUST: design of randomized trial of ultrasound-enhanced thrombolysis for acute ischemic stroke. J Neuroimaging 2004; 14: 108-12.

61. Bose A, Henkes H, Alfke K, et al.; Penumbra phase I stroke trial investigators. JNR Am J Neuroradiol 2008; 29: 1409-13.

62. Arnold M, Nedeltchev K, Schroth G, et al. Clinical and radiological predictors of recanalization and outcome of 40 patients with acute basilar artery occlusion treated with intra-arterial thromoblysis. J Neurol Neurosurg Psychiatry 2004; 75: 857-62.

63. Saqqur M, Uchino K, Demchuk AM, et al. Site of arterial occlusion identified by transcranial Doppler predicts the response to intravenous thrombolysis for stroke. Stroke 2007; 38: 948-54.

64. Linfante I, Llinas RH, Selim M, et al. Clinical and vascular outcome in internal carotid artery versus middle cerebral artery occlusions after intravenous tissue plasminogen activator. Stroke 2002; 33: 2066-71.

65. Flint AC, Cuckwiler GR, Budzik W, et al. Mechanical thrombectomy of intracranial internal carotid occlusion: pooled results of the $\mathrm{MERCI}$ and Multi MERCI Part I trials. Stroke 2007; 38: 1274-80.

66. Sorensen AG, Buonanno FS, Gonzalez RG, et al. Hyperacute stroke: evaluation with combined multi section diffusion-weighted and hemodynamically weighted echo-planar MR imaging. Radiology 1996; 199: 391-401.

67. Warach S, Dashe JF, Edelman RR. Clinical outcome in ischemic stroke predicted by early diffusion-weighted and perfusion magnetic resonance imaging: a preliminary analysis. J Cereb Blood Flow Metab 1996; 16: 53-9.

68. Barber PA, Darby DG, Desmond PM, et al. Prediction of stroke outcome with echoplanar perfusion and diffusion weighted MRI. Neurology 1998; 51: 418-26.

69. Tong DC, Yenari MA, Albers GW, O’Brien M, Marks MP, Moseley ME. Correlation of perfusion and diffusion weighted MRI with NIHSS score in acute ( $<6.5$ hour) ischemic stroke. Neurology 1998; 50: 864-70.

70. Kidwell CS, Alger JR, Di Salle F, et al. Diffusion MRI in patients with transient ischemic attacks. Stroke 1999; 30: $1174-80$.

71. Kidwell CS, Saver JL, Mattiello J, et al. Thrombolytic reversal of acute human cerebral ischemic injury shown by diffusion/perfusion magnetic resonance imaging. Ann Neurol 2000; 47: 462-9.

72. Chalela JA, Ezzeddine MA, Calabrese TM, et al. Diffusion and perfusion changes two hours after intravenous rt-PA therapy: a preliminary report. Stroke 2002; 33: 356-7.

73. Schacfer PW, Hussan Khani A, Christopher R, et al. Partial reversal of DWI abnormalities in stroke patients undergoing thrombolysis: evidence of DWI and ADC thresholds. Stroke 2002; 33: 357.

74. Neumann-Haefelin T, Kastrup A, de Crespigny A, et al. Serial MRI after transient focal cerebral ischemia in rats: dynamics of tissue injury, blood-brain barrier damage, and oedema formation. Stroke 2000; 31: 1965-72.

75. Fiehler J, Foth M, Kucinski T, et al. Severe ADC decreases do not predict irreversible tissue damage in humans. Stroke 2002; 33: 79-86.

76. Jansen O, Schellinger PD, Fiebach JR, Hacke W, Sartor K. Early recanalizations in acute ischemic stroke saves tissue at risk defined by MRI. Lancet 1999; 353: 2036-7. 
77. Schlaug G, Benfiled A, Baird AE, et al. The ischemic penumbra: operationally defined by diffusion and perfusion MRI. Neurology 1999; 53: 1528-37.

78. Schellinger PD, Jansen O, Fiebach JB, et al. Monitoring intravenous recombinant tissue plasminogen activator thrombolysis for acute ischemic stroke with diffusion and perfusion MRI. Stroke 2000; 31: 1318-28.

79. Schellinger PD, Jansen O, Fiebach JB, et al. Feasibility and practice of MR imaging of stroke in the management of hyperacute cerebral ischemia. AJNR Am J Neuroradiol 2000; 21: 1184-9.

80. Parsons MW, Yang Q, Barber PA, et al. Perfusion magnetic resonance imaging maps in hyperacute stroke: relative cerebral blood flow most accurately identifies tissue destined to infarct. Stroke 2001; 32: 1581-7.

81. Rordorf G, Koroshetz WJ, Copen WA, et al. Regional ischemia and ischemic injury inpatients with acute middle cerebral artery stroke as defined early diffusion-weighted and perfusion-weighted MRI. Stroke 1998; 29: 939-43.

82. Marks MP, Tong D, Beaulieu C, Albers G, deCrespigny A, Moseley ME. Evaluation of early reperfusion and IV rt-PA therapy using diffusion and perfusion weighted MRI. Neurology 1999; 52: 1792-8.

83. Albers GW, Thijs VN, Wechsler LR, et al. Magnetic resonance imaging profiles predict clinical response to early reperfusion: the diffusion and perfusion imaging evaluation for understanding stroke evolution (DEFUSE) study. Ann Neurol 2006; 60: 508-17.

84. Schellinger PD, Thomalla G, Fielhler J, et al. MRI-based and CT-based thrombolytic therapy in acute stroke within and beyond established time windows. An analysis of 1210 patients. Stroke 2007; 38: 2640-5. 